\title{
Recurrent intestinal metaplasia at the gastro- esophageal junction following endoscopic eradication of dysplastic Barrett's esophagus may not be benign
}

Authors

Institutions
Georgina R. Cameron ${ }^{1,3}$, Paul V. Desmond ${ }^{1,3}$, Chatura S. Jayasekera ${ }^{1,2,3}$, Francesco Amico ${ }^{2,3}$, Richard Williams ${ }^{1,3}$, Finlay A. Macrae ${ }^{2,3}$, Andrew C. F. Taylor ${ }^{1,3}$

${ }^{1}$ St Vincent's Hospital Melbourne, Melbourne, Victoria, Australia

${ }^{2}$ The Royal Melbourne Hospital, Melbourne, Victoria, Australia

${ }^{3}$ University of Melbourne, Melbourne, Victoria, Australia submitted

16. December 2015

accepted after revision

23. May 2016

Bibliography

DOI http://dx.doi.org/

10.1055/s-0042-109608

Published online: 9.8.2016

Endoscopy International Open

2016; 04: E849-E858

(c) Georg Thieme Verlag KG

Stuttgart · New York

E-ISSN 2196-9736

Corresponding author

\section{Georgina R. Cameron, MBBS}

(Hons) BMus (Hons)

St Vincent's Hospital Melbourne

- Gastroenterology

Level 4

Daly Wing

41 Victoria Parade

Fitzroy

Melbourne

Victoria 3065

Australia

georgiecameron@gmail.com
Fax: +61-3-92883590

Background and study aims: Radiofrequency ablation (RFA) combined with endoscopic mucosal resection (EMR) is effective for eradicating dysplastic Barrett's esophagus. The durability of response is reported to be variable. We aimed to determine the effectiveness and durability of RFA with or without EMR for patients with dysplastic Barrett's esophagus.

Patients and methods: Patients with dysplastic Barrett's esophagus referred to two academic hospitals were assessed with high definition whitelight endoscopy, narrow-band imaging, and Seattle protocol biopsies. EMR was performed in visible lesions. RFA was performed at 3-month intervals until complete remission of dysplasia (CR-D) and intestinal metaplasia (CR-IM) was achieved. Results: In total, 137 patients received RFA (78 with EMR); 75 with over 12 months follow-up since commencing RFA. Pretreatment histology was intramucosal cancer (IMC) $21 \%$, high grade

\section{Introduction}

\section{$\nabla$}

The AIM dysplasia trial, a randomized sham controlled trial published in 2008, showed that radiofrequency ablation (RFA) was highly effective in eradicating dysplastic Barrett's esophagus, and safe [1]. Subsequent studies have shown that combination endoscopic therapy (CET) (endoscopic mucosal resection (EMR) and RFA) provides a credible alternative to surgery in patients with early esophageal adenocarcinoma (EAC) [2-5]. EAC with submucosal invasion is still typically referred for esophagectomy due to risk of lymph node spread, which is reported to be between $10 \%$ and $50 \%$ depending on depth of mucosal invasion in the index EMR specimen [6-8]. RFA is now widely considered to be the preferred method for eradicating intestinal metaplasia and dysplasia within Barrett's esophagus [9].
While many studies report the efficacy of RFA in dysplastic Barrett's esophagus, there are fewer dysplasia (HGD) 54\%, low grade dysplasia (LGD) $25 \%$. CR-D rates were $88 \%, 92 \%$, and $100 \%$ at 1,2 , and 3 years; CR-IM rates were $69 \%, 74 \%$, and $81 \%$. Kaplan-Meier analysis showed increasing probability of achieving CR-D/CR-IM over time. Of 26 patients maintaining CR-IM for $>12$ months, five relapsed with intestinal metaplasia (19\%), and three with dysplasia (12\%). Recurrences occurred in patients with prior HGD/IMC, predominantly at the gastroesophageal junction (GEJ). None relapsed with cancer. Adverse events occurred in $4 \%$ of RFA and $6.5 \%$ of EMR procedures.

Conclusions: RFA combined with EMR is effective in achieving CR-D/CR-IM in the majority of patients with dysplastic Barrett's esophagus, with an incremental response over time. While durable in the majority, recurrent intestinal metaplasia and dysplasia, frequently occurring at the GEJ, suggest long-term surveillance is warranted in high risk groups.

published data on the durability of treatment outcomes. A systematic review and meta-analysis of the efficacy and durability of RFA in Barrett's esophagus [10] analyzed 18 efficacy and six durability studies. The pooled recurrence rate of intestinal metaplasia after eradication was estimated at $13 \%$ (95\%CI $9-18 \%$ ) [10]. Outcomes of more recent studies continue to vary. Phoa et al. reported outcomes of 54 patients receiving RFA and EMR for Barrett's esophagus with high grade dysplasia (HGD)/cancer and found sustained remission of intestinal metaplasia and neoplasia in $93 \%$ of those reaching 5 years of follow-up $(n=46)$ [11]. Others quote intestinal metaplasia recurrence rates between $20 \%$ and $32 \%[12,13]$.

Recurrent or residual intestinal metaplasia typically occurs in distinct patterns: as visible islands in the tubular esophagus; buried intestinal metaplasia under neo-squamous mucosa; and at the gastroesophageal junction (GEJ). Korst et al. found the GEJ was the most common site for recurrent 
intestinal metaplasia post-ablation (71\%) [12]. The clinical significance of intestinal metaplasia at the GEJ is not well established. Several clinical trials assessing RFA efficacy did not routinely biopsy the GEJ at follow-up $[1,14]$, partly accounting for the variation in quoted recurrence rates. In two studies where biopsies were routinely taken just below the neo-squamocolumnar junction post-RFA, one found persistent intestinal metaplasia in only $4 \%$ of 54 patients and no dysplasia; the other found recurrent dysplasia in $9 \%$ of 47 patients, with all dysplasia located at the GEJ $[11,13]$. With intestinal metaplasia thought to precede dysplasia, this suggests that intestinal metaplasia at the GEJ may not be a benign entity.

\section{Aims \\ $\nabla$}

We aimed to assess the effectiveness and durability of RFA (with or without EMR) in patients with dysplastic Barrett's esophagus, by determining rates of complete remission of dysplasia (CR-D) and intestinal metaplasia (CR-IM), time to achieve these end points, number of RFA treatments required, and recurrence rates and location of intestinal metaplasia/dysplasia post-eradication. A secondary aim was to evaluate the safety of EMR and RFA.

\section{Methods \\ $\nabla$}

Patients referred to St Vincent's Hospital Melbourne and The Royal Melbourne Hospital between 2008 and September 2013 were entered prospectively into a central database. Patient demographics, referral endoscopy details, prior treatment, and pre-assessment histology were recorded. Referral histology was reviewed by an expert gastrointestinal pathologist (RW).

Patients underwent systematic assessment of their Barrett's segment with high definition (Olympus $\mathrm{H}-180$ ) endoscopes using high definition white-light endoscopy (HD-WLE) and narrowband imaging (NBI). Forty patients (early in cohort) were also assessed with confocal endomicroscopy (CEM) as part of a study assessing the accuracy of HD-WLE, NBI, and CEM in identifying HGD/EAC. This study showed that CEM was accurate in confirming suspected dysplasia/neoplasia seen with HD-WLE/NBI, but did not significantly add to HGD/EAC detection or clinical outcome [15]. CEM was not routinely used for subsequent patient assessments. Barrett's extent was documented according to the Prague classification [16] (Appendix 1). Mucosal abnormalities were characterized according to size, Paris Classification [17] (Appendix 2), and mucosal pattern (irregularity/loss) [18]. Biopsies were taken according to the Seattle Protocol (Appendix 3). Biopsies of mucosal irregularities were labeled according to location (centimeters from mouth, o'clock position in neutral scope position), to facilitate location at subsequent endoscopy. Initially, where a lesion was thought to harbor HGD or neoplasia, biopsies were taken with EMR performed a few weeks later. In later cases, EMR was often performed at initial assessment. Biopsies were assessed for presence of intestinal metaplasia and grade of dysplasia using the revised Vienna classification (intestinal metaplasia without dysplasia (non-dysplastic Barrett's esophagus), indefinite for dysplasia (IND), low grade dysplasia (LGD), HGD or cancer) $[19,20]$. EMR specimens were evaluated for infiltration depth, vertical resection margins, tumor differentiation or grade of dysplasia, and lymphatic/vascular invasion.
Patients' subsequent management depended on the most advanced histology after assessment and suitability for CET. Those with submucosal cancer (SMC) were referred for esophagectomy or chemoradiotherapy as endoscopic therapy was not considered definitively curative. In those with intramucosal cancer (IMC) or nodular HGD, EMR was performed until we were confident no cancer remained before commencing RFA.

In the treatment group, RFA was performed at 3-month intervals, unless delay occurred due to patient illness/social reasons. RFA was performed using the BARRXTM HALO system as previously described in the literature [21]. The HALO360 or HALO90 ablation catheters were used at the discretion of the endoscopist (AT, FM, CJ, GC) depending on case specifics. Typically, HALO360 was used initially, unless the segment was patchy or significant narrowing existed from prior EMR scarring. Where HALO360 was used, the GEJ was overlapped and treated with the balloon. Where HALO90 was employed, the GEJ was focally ablated. In most cases, the esophagus was flushed with N-acetyl-cysteine pretreatment. Patients received double-dose proton pump inhibitor and topical anesthetic post-procedure. Interval EMR was performed where suspicious lesions were detected at subsequent endoscopy.

Follow-up evaluation commenced at the first post-treatment endoscopy confirming CR-IM (no endoscopic Barrett's and no intestinal metaplasia histologically, including at the GEJ). Recurrence was defined as intestinal metaplasia or dysplasia identified after achieving CR-IM. Intestinal metaplasia at the GEJ but not within the cardia was considered recurrence. Surveillance endoscopies were performed at 3, 6 and 12 months, then annually for those with prior IMC/HGD, and at 6 and 12 months, then annually for those with prior LGD. Surveillance biopsies were taken in four quadrants every $2 \mathrm{~cm}$ commencing just distal to the neosquamocolumnar junction at the GEJ, extending proximally for the original maximal (M) length of the segment.

Where possible, patients were followed in the tertiary center for at least 1 year after achieving CR-IM, however, in some cases, due to social circumstances or distance from the hospital, patients were discharged to their referring endoscopist. Advice was given with regard to surveillance intervals and biopsy protocol. Subsequent endoscopy/histology reports were obtained for database entry. Patients with recurrent intestinal metaplasia or dysplasia were re-referred to the tertiary center for further management. EMR and RFA adverse events were defined as events requiring surgery, unplanned hospital admission, bleeding requiring transfusion or unplanned endoscopic procedure. For patients living remotely, admission for observation was arranged when minor intraprocedural bleeding occurred. These patients were included in adverse event rates.

\section{Statistical analysis}

Absolute remission rates and Kaplan-Meier estimates with 95\% confidence intervals for achieving CR-D and CR-IM at 1, 2, and 3 years were calculated. Intestinal metaplasia/dysplasia recurrence rates (absolute, and Kaplan-Meier estimates) were calculated for those achieving CR-IM. Testing for statistical significance was performed using Chi squared/Fisher's exact test at a single tail significance level of $5 \%$.

This study was registered and approved by the hospitals' Human Research Ethics Committees. 


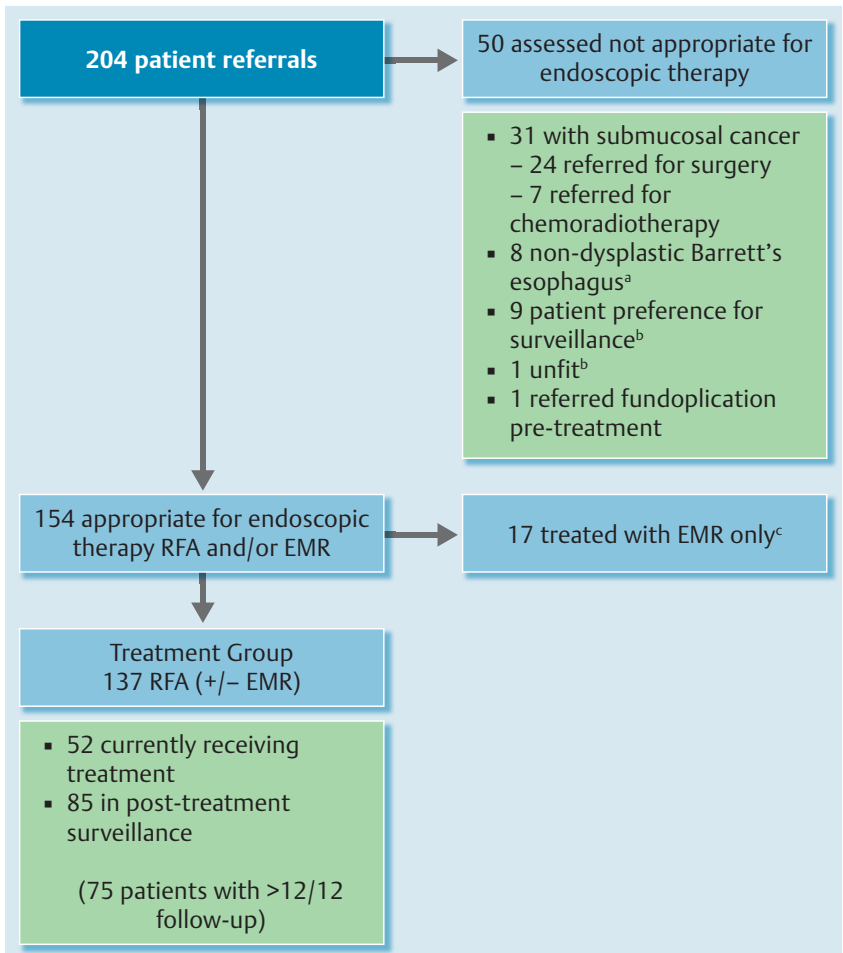

a Patients were referred with dysplasia but, following histology review and assessment, were found to have non-dysplastic Barrett's esophagus.

b Comorbidities of the patient unfit for therapy and five LGD patients that opted for surveillance, included, but were not limited to, significant cardiovascular and airways disease.

Compared with the treatment group, those who received EMR only had on average shorter $C$ and $M$ length of Barrett's $(P<0.001)$ and there was a trend toward more IMC as the pre-treatment histology though this was not statistically significant $(P=0.06)$.

Fig. 1 Treatment outcomes of patient referrals: Of 204 patients assessed, 137 have received RFA $\pm E M R$.

\section{Results}

$\nabla$

There were 204 patient referrals (170 males; median age 67.7 years [31.2-89.6]). Following assessment, 50 patients were excluded from receiving CET predominantly due to advanced disease; 24 with SMC were referred for esophagectomy, and seven for chemoradiotherapy. Of 154 patients appropriate for CET, 17 were treated with EMR only. $\bullet$ Fig. 1 details the referral cohort.

\section{Treatment group characteristics}

In total, 137 patients (118 males) had commenced RFA treatment at time of analysis. Pretreatment histopathology after full assessment was IMC in 29 (21\%), HGD in 74 (54\%) and LGD in 34 (25\%). Full assessment was defined as assessment endoscopy \pm EMR and referral histology review. Median age was 65.9 years (34.987.8); median Barrett's esophagus circumferential (C) and $M$ length were $3 \mathrm{~cm}(0-17)$ and $5 \mathrm{~cm}(0-18)$, respectively. In total, $78 / 137$ patients had EMR in addition to RFA (69 pre-RFA, three between treatments, six post-RFA); 38/137 had received Barrett's esophagus treatment before referral: 22 with EMR, six argon plasma coagulation (APC), three laser, two cryotherapy, two photodynamic therapy, one gold probe, and two chemoradiotherapy (one for squamous cell carcinoma (SCC) of the esophagus).

\section{Effectiveness}

At the time of census, 85/137 were in post-treatment surveillance (five having achieved CR-D but not CR-IM), and 75 had at least 12 months of follow-up from first RFA to last endoscopy (therapeutic or surveillance). Of those with 12 months follow-up, nine had received HALO360 only (35 with HALO360/90, 31 with HALO90). In total, 90 patients achieved CR-D (median time 7.2 months [2.341.6]); 65 achieved CR-IM (median 8.6 months [2.3 - 30.5]). Median number of RFA to achieve these end points was $2(1-6)$. Median follow-up from first RFA to last endoscopic procedure was 13 months (0-53.7). Absolute rates of CR-D and CR-IM for those with 1-, 2-, and 3-year follow-up were $88 \%$, $92 \%$, and $100 \%$, and $69 \%, 74 \%$, and $81 \%$, respectively. Kaplan-Meier estimates for the same end points at 1,2 , and 3 years were $58 \%, 88 \%$, and $95 \%$, and $41 \%, 72 \%$, and $82 \%$. These results, with associated $95 \%$ confidence intervals are summarized in $\bullet$ Table 1 and $\bullet$ Fig. 2 a, $\bullet$ Fig. $\mathbf{2 b}$. Of the 75 patients with at least 12 months of follow-up, at time of census, nine had not yet achieved CR-D (median follow-up 18.4 months [12.4-35.3]), and 24 had not yet achieved CR-IM (median follow-up 19.5 months [12.0-58.9]), both groups with median 3 RFA (1-7). Of those not yet achieving CR-D, two were discharged from follow-up for social reasons without histological confirmation of CR-D. Two were awaiting follow-up biopsies. One was still receiving RFA after several intervening EMR procedures for nodular Barrett's esophagus. Two had a poor endoscopic response to RFA thought due to visible volume reflux and were referred for fundoplication before continuing treatment. Two were referred for esophagectomy after cancer was found during RFA treatment. In one case, the pre-assessment histology was HGD with non-dysplastic Barrett's esophagus found at referral and assessment. Three months following initial HALO90, a nodule harboring SMC was detected and completely excised with EMR. No residual cancer was found in the esophagectomy specimen. The second patient had IMC documented previously but HGD at referral and assessment. No EMR was performed. IMC was subsequently detected 3 months after two HALO360. Esophagectomy was curative. Five-year follow-up showed no endoscopic recurrence in either case. Of the 24 patients not yet achieving CR-IM, four had no visible Barrett's esophagus, seven had $<1 \mathrm{~cm}$ Barrett's esophagus, 13 had $>1 \mathrm{~cm}$ Barrett's esophagus remaining. Location of intestinal metaplasia was focal at the GEJ in five, focal above the GEJ in four, diffuse in six patients, not specified in six, and in three there were no biopsies.

There was no statistically significant difference in response to therapy based on pretreatment histology.

\section{Durability}

In total, 65 patients achieved CR-IM (median follow-up 12.4 months $(0-64.4)$ ). Of these, 12/65 had "possible Barrett's" described at endoscopy (eight with tiny islands, one with possible rim at GEJ, three with possible Barrett's tongues of $\sim 1 \mathrm{~cm}$ ) but targeted biopsies of these areas did not reveal intestinal metaplasia. In total, 26 patients had at least 12 months follow-up after achieving CR-IM. Of those achieving CR-IM, five did so after one HALO360 (one with EMR also); three of those had at least 12 months of follow-up since achieving CR-IM; 18/65 (28\%) patients documented recurrent intestinal metaplasia after initial eradication, $72 \%$ at the GEJ; 10/18 had salvage therapy (APC 5, EMR 3, RFA 2). Nine had re-achieved CR-IM at time of census. Of the nine re-achieving CR-IM, median months in remission was 12.8 $(0-38.9)(\bullet$ Fig. 3a, $\bullet$ Table 2). 


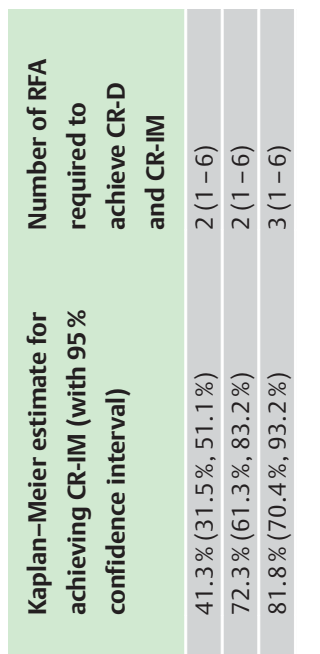

In total, 6/65 (9\%) patients documented recurrent dysplasia after achieving CR-IM, $80 \%$ at the GEJ. Five patients subsequently reachieved CR-D, two with salvage therapy (RFA plus EMR), and in three, dysplasia was not detected at subsequent endoscopies during 17, 25, and 39 months of follow-up ( $\bullet$ Fig. $3 \mathbf{b}$, $\odot$ Table 2).

Of those patients maintaining CR-IM for at least 12 months ( $\mathrm{n}=$ 26), recurrent intestinal metaplasia and dysplasia occurred in five (19\%) and three (12\%), respectively. The Kaplan-Meier estimate of durability of CR-IM at 1 year was $64 \%$ (95\%CI $49-59 \%$ ), and at 2 and 3 years, it was $48 \%$ (95\%CI 29-67\%) (- Fig.4); for CR-D, the estimates were $88 \%$ ( $80-97 \%), 84 \%$ (73-96\%), and $77 \%(61-94 \%)$ at 1,2 , and 3 years, respectively.

In all cases with recurrent intestinal metaplasia/dysplasia, the most advanced pretreatment histopathology was HDG or IMC. In total, $13 / 18$ intestinal metaplasia recurrences were in one biopsy only and $13 / 18$ were at the GEJ; nine re-achieved CR-IM. Dysplasia recurrence was HGD $(n=4)$ and LGD $(n=2)$ with no EAC; 5/6 recurrences were focal at the GEJ; one had buried dysplasia, five subsequently re-achieved CR-IM. There was no statistically significant difference in recurrence rates between those who received HALO9O at the GEJ, and those who received HALO360 alone. Additionally, there was no statistically significant difference in recurrence between those with possible Barrett's at endoscopy and those without.

\section{Safety of RFA and EMR}

In the treatment group, 137 patients had RFA (305 procedures) and 78 had EMR (122 procedures). Adverse events occurred in $4 \%$ of RFA and $6.5 \%$ of EMR procedures. In the entire referral cohort, 215 EMR procedures were performed in 140 patients, with a $9 \%$ adverse event rate. Adverse event details are summarized in - Table 3.

\section{Discussion}

We present a case series of patients with dysplastic Barrett's esophagus treated with RFA (with or without EMR). While RFA has been established as safe and effective for treating dysplastic Barrett's esophagus, there is relative paucity in the literature with regard to outcome durability. Our data are a significant addition to the growing literature on recurrence rates of intestinal metaplasia and dysplasia in a histologically advanced group. Our data show that where intestinal metaplasia and dysplasia recurred, it did so most frequently at the GEJ and in those with prior advanced histology. Our data also show an incremental response to RFA over time, with continued treatment.

\section{Effectiveness}

Our CR-IM and CR-D rates at 1, 2, and 3 years were $69 / 74 / 81 \%$, and $88 / 92 / 100 \%$, respectively with lower Kaplan-Meier estimates for the same time points. The absolute rates are similar to those published in the literature though on the lower end of the spectrum. The pooled percentage of patients achieving CR-IM and CR-D in Orman et al.'s systematic review (2013) was 78\% (95\%CI, 70-86\%) and $91 \%$ (95\%CI, 87-95\%), respectively [10]. Duration of study follow-up was variable with a median of 20.5 months (12-31 months).

While many studies show efficacy rates for RFA after a given time, studies have not described remission rates improving over time with continued treatment. Kaplan-Meier analysis of our cohort showed that the probability of achieving CR-D/CR-IM improved 


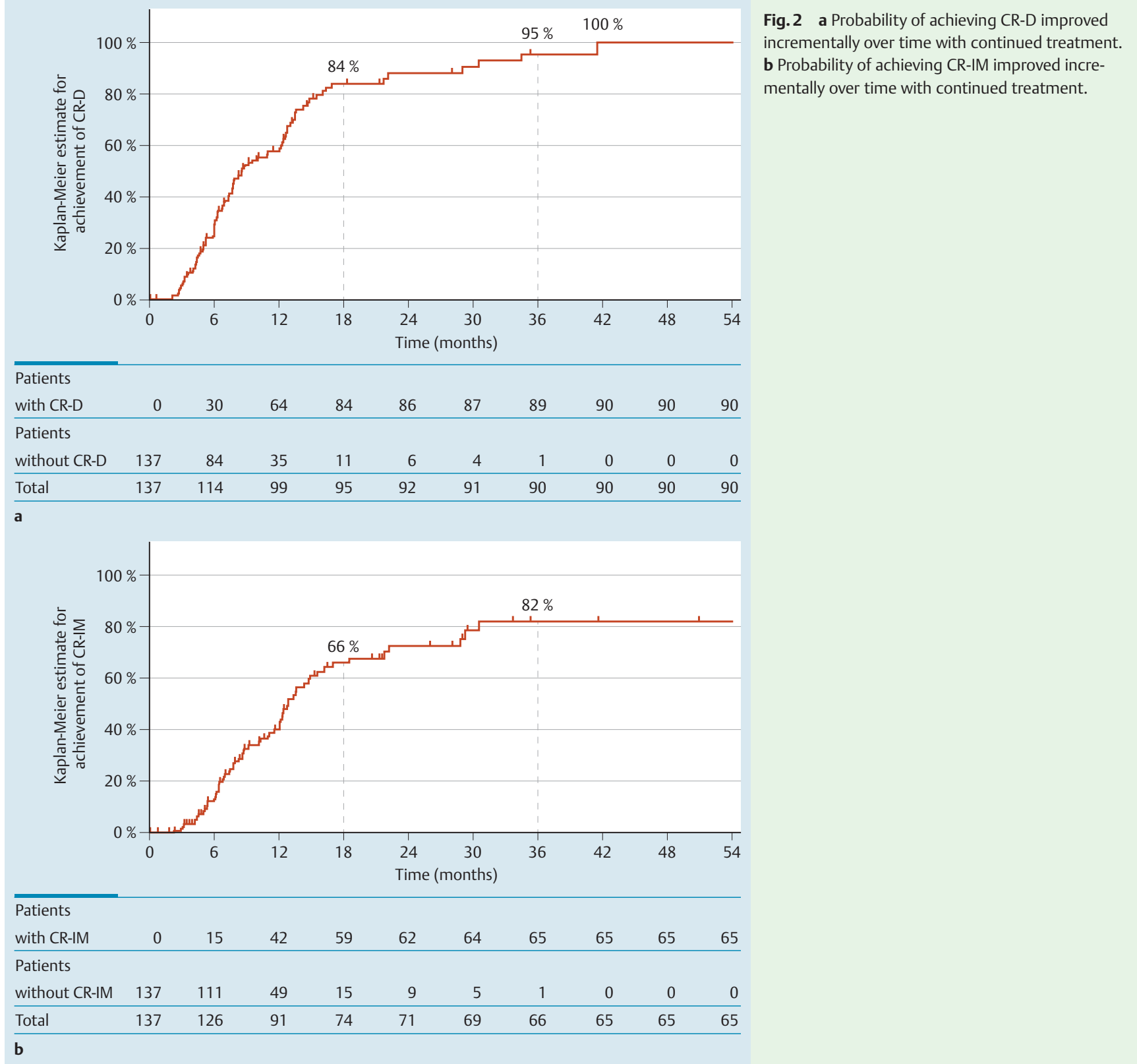

incrementally over time with further RFA treatments: $84 \%$ CR-D/ 66\% CR-IM within 18 months, and 95\% CR-D/82\% CR-IM within 36 months ( Fig. 2a, $\bullet$ Fig. 2 b). It is likely that patients with complex Barrett's esophagus segments (i.e. nodular, strictured, scarred or patulous esophagus) require more RFA sessions to achieve complete segment ablation due to technical difficulty in achieving total ablation at each session. A second subgroup of "slow responders" may exist who, despite complete ablation in a session, require a greater number of treatments to achieve CR-IM. We hypothesize that this group may include those with poorly controlled volume reflux resulting in ongoing mucosal insult between treatments. Further evaluation of our cohort is required to determine what proportion of patients yet to achieve CR-D/CRIM respond eventually. Further studies are needed to assess predictors of poor/slow response.

Our cohort was heterogeneous, reflecting the broad spectrum of patients with dysplastic Barrett's esophagus, and consisted of patients with predominantly advanced histology (>75\% with HGD/IMC). Cases included often complex Barrett's esophagus segments and this was thought to contribute to slightly lower CR-D/CR-IM rates at early time points. Delay between RFA treatments due to medical/social reasons, or interval performance of EMR for suspicious lesions were also factors. Finally, 3/24 patients not achieving CR-IM and 4/9 patients not achieving CR-D after 12 months of treatment lacked histological confirmation at the time of census. It is therefore conceivable that the proportion of patients achieving CR-D/CR-IM is an underestimate.

\section{Progression of disease}

EAC was detected in two patients while receiving RFA, resulting in referral for esophagectomy (details in Results section). In both cases, given the time course, cancer likely went undetected at assessment rather than representing true progression on treatment. These cases highlight the importance of rigorous baseline evaluation to enable appropriate patient selection for CET. Both cases occurred very early in the cohort suggesting that a learning curve exists, even for experienced endoscopists, in recognizing subtle mucosal abnormalities that may harbor dysplasia or can- 


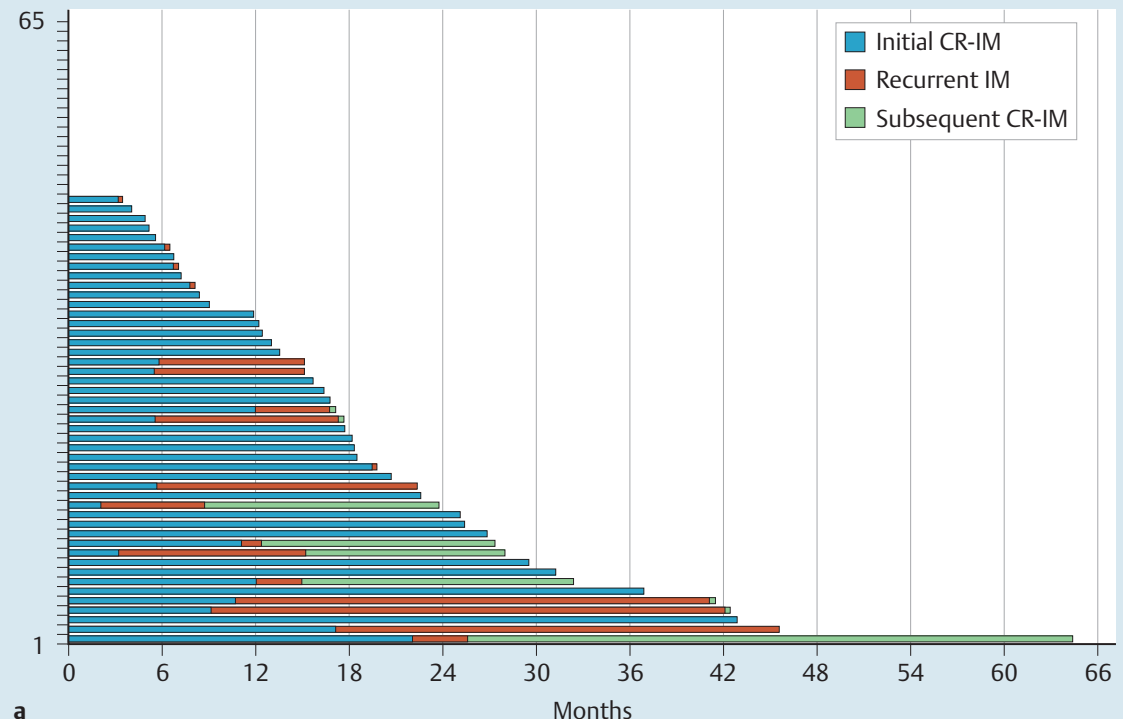

Fig. 3 a Experience of 65 patients initially achieving CR-IM, showing recurrence of intestinal metaplasia and subsequent remission: Of 65 patients achieving CR-IM, 18 had recurrent intestinal metaplasia with nine of those re-achieving CR-IM.

b Experience of 65 patients initially achieving CR-IM, showing recurrence of dysplasia and subsequent remission: Of 65 patients achieving CR-IM, six had recurrent dysplasia with five of those re-achieving CR-D and CR-IM.
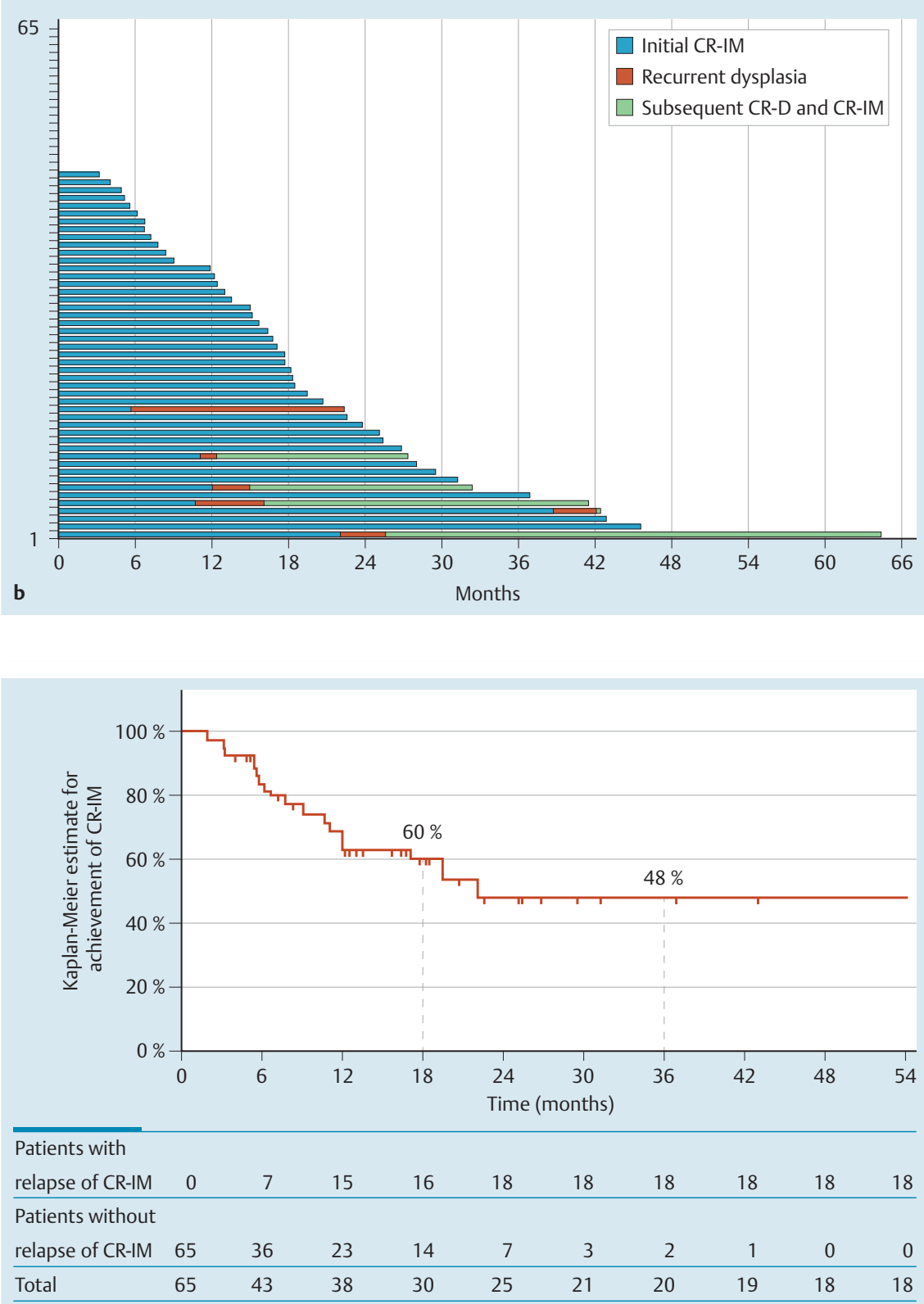

Fig. 4 Durability of CR-IM over time. 


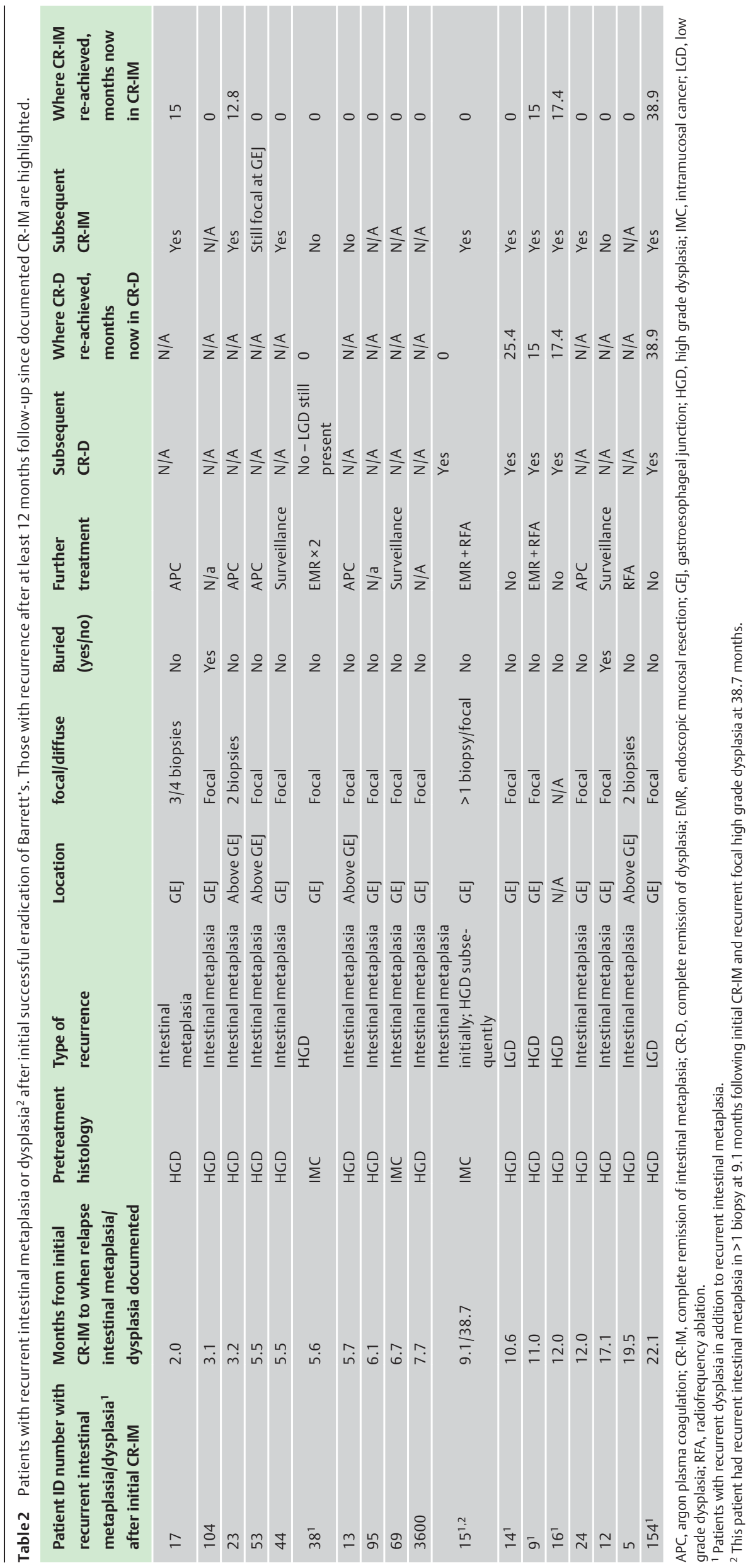


Table 3 Adverse events for RFA and EMR. Section a outlines the adverse event rate for RFA procedures and Section b outlines the adverse event rate for EMR procedures in those patients who also underwent RFA treatment (i.e. had combined endoscopic therapy). Section c outlines the EMR adverse event rate for the entire referral cohort, whether they had EMR for staging, sole treatment or as part of combination endoscopic therapy and thus includes patients from Sections $a$ and $b$.

\begin{tabular}{|c|c|c|c|c|c|}
\hline & Number & Type of adverse events & Number & $\%$ & Detail \\
\hline \multicolumn{6}{|c|}{ a: RFA complications of the treatment cohort (RFA $\pm E M R)$} \\
\hline Patients undergoing RFA & 137 & & & & \\
\hline Total RFA Procedures & 305 & & & & \\
\hline Procedures per patient & Median $2(1-7)$ & & & & \\
\hline \multirow[t]{8}{*}{ Total adverse events } & $12(4 \%)$ & Mucosal tears & 2 & $1.3 \%$ & Secondary to sizing balloon \\
\hline & & & 2 & & RFA 360 balloon \\
\hline & & $\begin{array}{l}\text { Bleeding - requiring } \\
\text { transfusion }\end{array}$ & 3 & $0.9 \%$ & $\begin{array}{l}\text { Required transfusion and repeat endoscopic } \\
\text { procedure (one in setting of re-warfarinization }\end{array}$ \\
\hline & & $\begin{array}{l}\text { Bleeding-admitted } \\
\text { for observation }\end{array}$ & 1 & $0.3 \%$ & Admitted for observation \\
\hline & & Stricture & 4 & $1.3 \%$ & 2 requiring $\geq 2$ dilations \\
\hline & & & & & 2 requiring single dilation \\
\hline & & Fever $(0.3 \%)$ & 1 & $0.3 \%$ & Admitted for observation \\
\hline & & Pain $(0.3 \%)$ & 1 & $0.3 \%$ & Admitted for observation \\
\hline \multicolumn{6}{|c|}{ b: EMR complications occurring within the treatment cohort only (EMR + RFA) } \\
\hline Patients & 78 & & & & \\
\hline Procedures & 122 & & & & \\
\hline Procedures per patient & Median $1(1-8)$ & & & & \\
\hline \multirow[t]{6}{*}{ Adverse events } & $8(6.5 \%)$ & Perforation & 1 & $0.8 \%$ & EMR of IMC at GEJ \\
\hline & & & & & Laparotomy and oversew \\
\hline & & $\begin{array}{l}\text { Bleeding - requiring } \\
\text { transfusion }\end{array}$ & 2 & $1.6 \%$ & 1 secondary to Mallory Weiss tear \\
\hline & & $\begin{array}{l}\text { Bleeding-admitted } \\
\text { for observation }\end{array}$ & 3 & $2.5 \%$ & Admitted overnight \\
\hline & & Stricture & 1 & $0.8 \%$ & Requiring $\geq 2$ dilations \\
\hline & & Fever & 1 & $0.8 \%$ & Admitted for observation \\
\hline \multicolumn{6}{|c|}{ c: EMR complications occurring in the entire referral cohort (EMR at any time point) } \\
\hline Patients & 140 & & & & \\
\hline Procedures & 215 & & & & \\
\hline Procedures per patient & Median $1(1-8)$ & & & & \\
\hline \multirow[t]{8}{*}{ Adverse events } & $20(9 \%)$ & Perforation & 1 & $0.5 \%$ & EMR of IMC at GEJ \\
\hline & & & & & Laparotomy and oversew \\
\hline & & Suspected perforation & 4 & $1.8 \%$ & $\begin{array}{l}\text { Clipped } \\
\text { Perforation excluded }\end{array}$ \\
\hline & & $\begin{array}{l}\text { Bleeding - requiring } \\
\text { transfusion }\end{array}$ & 2 & $0.9 \%$ & 1 secondary to Mallory Weiss tear \\
\hline & & $\begin{array}{l}\text { Bleeding-admitted } \\
\text { for observation }\end{array}$ & 8 & $3.7 \%$ & Admitted overnight \\
\hline & & Stricture & 2 & $1.4 \%$ & Requiring $\geq 2$ dilations \\
\hline & & & 1 & & Single dilation \\
\hline & & Fever & 2 & $0.9 \%$ & Admitted for observation \\
\hline
\end{tabular}

EMR, endoscopic mucosal resection; GE], gastroesophageal junction; IMC, intramucosal cancer; RFA, radiofrequency ablation.

cer. This is supported by findings published on a subset of our cohort that found that Barrett's assessment in specialized Barrett's units resulted in improved detection of mucosal abnormalities and EAC [22].

\section{Durability}

Our data show that, in patients achieving CR-IM, there was a significant risk of recurrent intestinal metaplasia (28\%) and dysplasia $(9 \%)$. Those documenting recurrence before 12 (and even 6) months ( $\bullet$ Fig. 3 a) raise the possibility of the initial CR-IM diagnosis resulting from biopsy sampling error. Intestinal metaplasia and dysplasia recurrence rates in patients with at least 12 months in remission (possibly better representatives of true remission) were still $19 \%$ and $12 \%$, respectively. These figures may be underestimates as a small number of patients achieving CR-IM were referred back to their original endoscopist for surveillance (as outlined in the Methods section). Based on receipt of patients' subsequent endoscopy/histology reports, we believe that protocol was well adhered to outside the tertiary center and that potential classification bias was minimized.

There is currently inconsistency in the literature with regard to intestinal metaplasia recurrence, partly due to lack of consensus in its definition. A US Multicenter Consortium, whose definition included isolated intestinal metaplasia of the cardia/GEJ, found that, among patients achieving CR-IM by 24 months, 20\% developed recurrent intestinal metaplasia within a year, and $33 \%$ after 2 years. Intestinal metaplasia of the cardia/GEJ accounted for almost half of the recurrences reported [23]. Korst et al. described recurrent intestinal metaplasia in $26 \%$ of 53 patients treated successfully with RFA. None relapsed with dysplasia though this 
cohort consisted of predominantly non-dysplastic Barrett's esophagus [12]. Vaccaro et al. found 26\% cumulative intestinal metaplasia recurrence in 47 patients treated successfully with RFA [13]. This group had predominantly HGD pretreatment, though it included non-dysplastic Barrett's esophagus and IMC. Four $(9 \%)$ had recurrent dysplasia at the neo-squamocolumnar junction; none were detected endoscopically. In Phoa et al.'s cohort, biopsies were obtained from the GEJ (median 20) which was focally ablated at least once [11]. Recurrent focal intestinal metaplasia was found in 19/54 patients (35\%), however, this finding was not reproduced at subsequent endoscopies in 17/19 patients (median of five follow-up endoscopies), with no increase in intestinal metaplasia incidence at the GEJ over time [11]. In contrast, where intestinal metaplasia of the GEJ was not considered recurrence, reported recurrence rates were much lower. Of 198 patients achieving CR-IM in a UK study, only $9 \%$ had recurrent intestinal metaplasia by the end of follow-up; $47 \%$ of those, however, had recurrent dysplasia [24]. Whether intestinal metaplasia at the GEJ represents true recurrence, persistent intestinal metaplasia in an area not initially ablated, or cardia intestinal metaplasia is uncertain.

Our intestinal metaplasia recurrence rates are in line with those studies routinely obtaining biopsies at the GEJ, as we did [11 - 13, 23]. All patients with recurrent dysplasia/intestinal metaplasia had HGD or IMC as their most advanced pretreatment histology. None recurred with a more advanced histology than previously documented, and importantly, none with recurrence developed cancer. Recurrences were predominantly focal, and were located at the GEJ in $72 \%$ with recurrent intestinal metaplasia and $82 \%$ with recurrent dysplasia. There was no statistically significant difference in recurrence rates between those who received HALO90 at the GEJ, and those who received HALO360 alone, however, the sample size of the latter group was small. The authors recognize that the lack of focal ablation of the z-line with HALO90 in a few patients may have impacted overall recurrence rates and advocate focal GEJ ablation in all patients where possible. All with recurrence were able to be managed endoscopically with the majority re-achieving CR-D/CR-IM.

The risk for intestinal metaplasia progression to dysplasia/neoplasia at the GEJ is also unclear. Our data show that where dysplasia recurred, it did so at the GEJ in $83 \%$; consistent with the finding of Vaccaro et al. [13]. Given that intestinal metaplasia precedes dysplasia, it follows that intestinal metaplasia at the GEJ, after RFA eradication of Barrett's esophagus, is not necessarily benign. Like Vaccaro et al., our data indicate that, following successful ablation of dysplastic Barrett's esophagus, the GEJ should be considered an area at risk for the development of dysplasia and potentially adenocarcinoma in high risk groups. It would suggest that the finding of intestinal metaplasia at the GEJ should not be excluded from reports of recurrence rates and durability.

Some early RFA cost analyses models incorporated the assumption that patients in whom dysplastic Barrett's esophagus was eradicated with RFA did not require long-term surveillance [25]. Later models are including evidence such as ours that the potential for intestinal metaplasia and dysplasia recurrence may necessitate ongoing surveillance, particularly in high risk groups [26]. Further dedicated studies are required assessing the significance of intestinal metaplasia at the GEJ post-ablation.

\section{Limitations of study}

This study was a retrospective analysis of the outcomes of combined endoscopic treatment in a real-world cohort of Barrett's patients. As previously mentioned, the cohort had predominantly advanced disease, with often complex segments, and several had received prior endoscopic treatment before their referral to a tertiary center. Decisions with regard to individual patient treatment were made at the discretion of the endoscopist, and while following recommended guidelines, adjustments to treatment were made when deemed clinically appropriate. These factors would have impacted CR-IM and CR-D rates. Additionally, the lack of focal ablation of the z-line with HALO90 in a few patients may have impacted overall recurrence rates and the authors advocate focal GEJ ablation in all patients where possible. Finally, this study reports the preliminary data set of a cohort that is being followed long term. At the time of census, there were only 16 patients who had reached at least 3 years of follow-up since first RFA and only 26 with at least 12 months follow-up since achieving the end point of CR-IM. The latter was in part due to some patients returning to their referring endoscopists for surveillance following CR-IM, again, a familiar scenario in real-world practice. We aim to reassess our durability outcomes in future analyses with a greater number of patients with longterm follow-up and additionally aim to recapture data for those patients previously returned to their referring specialist for surveillance.

\section{Conclusion}

RFA, combined with EMR in select patients, is effective, safe, and durable for the majority of patients with dysplastic Barrett's esophagus including those with complex disease. Dysplasia and intestinal metaplasia recurrence appears to occur more commonly in patients with advanced pretreatment histology, and frequently at the GEJ. This suggests that recurrent intestinal metaplasia at the GEJ may not be a benign finding and that ongoing careful surveillance of this region should be considered in patients with previous advanced dysplasia.

\section{Appendix 1 \\ $\nabla$}

Prague Classification [16]

A circumferential segment of Barrett's esophagus of $2 \mathrm{~cm}$ in length from the top of the mucosal folds and tongues of Barrett's metaplasia extending an additional $3 \mathrm{~cm}$ superior would be classified as C2M5.

- Circumferential $(\mathrm{C})$ length of Barrett's esophagus

- Maximal (M) extent of any Barrett's metaplasia. 


\section{Appendix 2}

$\nabla$

\begin{tabular}{|c|c|c|}
\hline \multicolumn{3}{|c|}{ Paris classification of mucosal lesions [17]. } \\
\hline $\begin{array}{l}\text { Endoscopic } \\
\text { appearance }\end{array}$ & $\begin{array}{l}\text { Paris } \\
\text { classification }\end{array}$ & Description \\
\hline Protruded lesions & 15 & Nodule, sessile polyp \\
\hline \multirow[t]{2}{*}{ Flat elevated lesions } & $02 \mathrm{~A}$ & Flat elevation of mucosa \\
\hline & $02 \mathrm{~A}+\mathrm{C}$ & $\begin{array}{l}\text { Flat elevation of mucosa } \\
\text { with central depression }\end{array}$ \\
\hline \multirow[t]{3}{*}{ Flat lesions } & 02B & Flat mucosal change \\
\hline & $02 \mathrm{C}$ & Mucosal depression \\
\hline & $02 \mathrm{C}+\mathrm{A}$ & $\begin{array}{l}\text { Mucosal depression } \\
\text { with raised edge }\end{array}$ \\
\hline
\end{tabular}

\section{Appendix 3}

Seattle Protocol [27]

Random 4-quadrant biopsies taken at 1- to 2-cm intervals along the Barrett's segment using jumbo biopsy forceps.

\section{Competing interests: None}

\section{References}

1 Shaheen NJ, Sharma P, Overholt BF et al. Radiofrequency ablation in Barrett's esophagus with dysplasia. NEJM 2009; 360: 2277-8888

2 Bulsiewicz WJ, Kim HP, Dellon ES et al. Safety and efficacy of endoscopic mucosal therapy with radiofrequency ablation for patients with neoplastic Barrett's esophagus. Clin Gastroenterol Hepatol 2013; 11: $636-642$

3 Okoro NI, Tomizawa Y, Dunagan KT et al. Safety of prior endoscopic mucosal resection in patients receiving radiofrequency ablation of Barrett's esophagus. Clin Gastroenterol Hepatol 2012; 10: 150-154

4 Pouw RE, Wirths K, Eisendrath $P$ et al. Efficacy of radiofrequency ablation combined with endoscopic resection for Barrett's esophagus with early neoplasia. Clin Gastroenterol Hepatol 2010; 8: 23-29

5 van Vilsteren FG, Pouw RE, Seewald S et al. Stepwise radical endoscopic resection versus radiofrequency ablation for Barrett's oesophagus with high-grade dysplasia or early cancer: a multicentre randomised trial. Gut 2011; 60: 765-773

6 Holscher $A H$, Bollschweiler E, Schroder $W$ et al. Prognostic impact of upper, middle, and lower third mucosal or submucosal infiltration in early esophageal cancer. Ann Surg 2011; 254: 802-807; discussion $7-8$

7 Luna RA, Gilbert E, Hunter JG. High-grade dysplasia and intramucosal adenocarcinoma in Barrett's esophagus: the role of esophagectomy in the era of endoscopic eradication therapy. Curr Opin Gastroenterol 2012; 28: $362-369$

8 Alvarez Herrero L, Pouw RE, van Vilsteren FG et al. Risk of lymph node metastasis associated with deeper invasion by early adenocarcinoma of the esophagus and cardia: study based on endoscopic resection specimens. Endoscopy 2010; 42: 1030-1036

9 Shaheen NJ, Overholt BF, Sampliner RE et al. Durability of radiofrequency ablation in Barrett's esophagus with dysplasia. Gastroenterology 2011; $141: 460-468$
10 Orman ES, Li N, Shaheen NJ. Efficacy and durability of radiofrequency ablation for Barrett's esophagus: Systematic review and meta-analysis. Clin Gastroenterol Hepatol 2013; 11: 1245-1255

11 Phoa KN, Pouw RE, van Vilsteren FG et al. Remission of Barrett's esophagus with early neoplasia 5 years after radiofrequency ablation with endoscopic resection: a Netherlands cohort study. Gastroenterology 2013; 145: $96-104$

12 Korst RJ, Santana-Joseph S, Rutledge JR et al. Patterns of recurrent and persistent intestinal metaplasia after successful radiofrequency ablation of Barrett's esophagus. J Thorac Cardiovasc Surg 2013; 145: $1529-1534$

13 Vaccaro BJ, Gonzalez S, Poneros JM et al. Detection of intestinal metaplasia after successful eradication of Barrett's Esophagus with radiofrequency ablation. Dig Dis Sci 2011; 56: 1996-2000

14 Fleischer DE, Overholt BF, Sharma VK et al. Endoscopic radiofrequency ablation for Barrett's esophagus: 5-year outcomes from a prospective multicenter trial. Endoscopy 2010; 42: 781 - 789

15 Jayasekera C, Taylor AC, Desmond PV et al. Added value of narrow band imaging and confocal laser endomicroscopy in detecting Barrett's esophagus neoplasia. Endoscopy 2012; 44: 1089-1095

16 Sharma P, Dent J, Armstrong D et al. The development and validation of an endoscopic grading system for Barrett's esophagus: the Prague C \& M criteria. Gastroenterology 2006; 131: 1392 -1399

17 Endoscopic Classification Review G. Update on the Paris classification of superficial neoplastic lesions in the digestive tract. Endoscopy 2005; 37: $570-578$

18 Sharma P, Weston AP, Topalovski M et al. Magnification chromoendoscopy for the detection of intestinal metaplasia and dysplasia in Barrett's oesophagus. Gut 2003; 52: 24-27

19 Schlemper RJ, Riddell RH, Kato Y et al. The Vienna classification of gastrointestinal epithelial neoplasia. Gut 2000; 47: 251-255

20 Schlemper RJ, Kato Y, Stolte M. Diagnostic criteria for gastrointestinal carcinomas in Japan and Western countries: proposal for a new classification system of gastrointestinal epithelial neoplasia. J Gastroenterol Hepatol 2000; 15 (Suppl 3): G49-G57

21 Pouw RE, Sharma VK, Bergman JJ et al. Radiofrequency ablation for total Barrett's eradication: a description of the endoscopic technique, its clinical results and future prospects. Endoscopy 2008; 40: 1033-1040

22 Cameron GR, Jayasekera CS, Williams $R$ et al. Detection and staging of esophageal cancers within Barrett's esophagus is improved by assessment in specialized Barrett's units. Gastrointest Endosc 2014; 80: 971-983.e1

23 Gupta M, Iyer PG, Lutzke $L$ et al. Recurrence of esophageal intestinal metaplasia after endoscopic mucosal resection and radiofrequency ablation of Barrett's esophagus: results from a US Multicenter Consortium. Gastroenterology 2013; 145: 79-86, e1

24 Haidry RJ, Dunn JM, Butt MA et al. Radiofrequency ablation and endoscopic mucosal resection for dysplastic Barrett's esophagus and early esophageal adenocarcinoma: outcomes of the UK National Halo RFA Registry. Gastroenterology 2013; 145: 87-95

25 Inadomi JM, Somsouk M, Madanick RD et al. A cost-utility analysis of ablative therapy for Barrett's esophagus. Gastroenterology 2009; 136: $2101-2114, \mathrm{e} 1-6$

26 Hur C, Choi SE, Rubenstein JH et al. The cost effectiveness of radiofrequency ablation for Barrett's esophagus. Gastroenterology 2012; 143: $567-575$

27 Spechler SJ, Sharma P, Souza RF et al. American Gastroenterological Association. American Gastroenterological Association technical review on the management of Barrett's esophagus. Gastroenterology 2011; 140: e18-52; quiz e13 